\title{
An Emotional Adaption Approach to increase Helpfulness towards a Robot
}

\author{
Barbara Gonsior $^{1,2}$, Stefan Sosnowski ${ }^{1}$, Malte Buß $\beta^{1}$, Dirk Wollherr ${ }^{1,2}$ and Kolja Kühnlenz ${ }^{1,2}$
}

\begin{abstract}
This paper describes a new methodological approach and robot system to trigger more prosocial human reactions towards a robot by transferring social-psychological principles from human-human interaction to human-robot interaction (HRI). The main idea is to trigger increased helpfulness by proactively creating similarity through dynamic emotional adaption of the robot to the mood of the human. This is achieved in an explicit and implicit way: Explicitly, by a similarity-statement of the robot of being in the same mood as the user, and implicitly by controlling the affective parameters of facial and verbal expressions of a robot head in an interaction scenario such that the current values of the human mood in the dimensions of pleasure, arousal, and dominance (PAD) are matched. In a first step, this is accomplished by an initial self-assessment by the human participant to be extended by automatic emotion recognition modules in a later stage. The effectiveness of the approach is confirmed by significant experimental results.
\end{abstract}

\section{INTRODUCTION}

With increasing demand for service robots to support human users by fulfilling tasks in human centered environments, research on human-robot interaction (HRI) gains more and more importance. Prominent application scenarios for such robots are manifold and reach from tour guide [1] and shopping robots [2] to home assistance and care [3].

In any interaction, emotions are an important issue. In 1995, Picard coined the term "Affective Computing" [4]. It describes a form of computing that "relates to, arises from, or influences emotions". Picard pointed out that this might lead to increased performance and decision making for the computer, stressing the importance of such ideas. Today, a large amount of works incorporate this idea. Two main aspects of affective computing are systems detecting emotions in the human user, and systems showing emotions themselves. The detection of emotions and its use

This work is supported in part by the EU FP7 STREP project "IURO - Interactive Urban Robot)," contract number 248317, see www.iuroproject.eu, the ERC Advanced Grant project "SHRINE - Seamless Human Robot Interaction in Dynamic Environments," contract number 267877, within the DFG excellence initiative research cluster Cognition for Technical Systems - CoTeSys, see www.cotesys.org, and by the Institute for Advanced Study (IAS), Technische Universität München, see also www.tum-ias.de. The authors like to thank Elokence (see www.elokence.com) for providing the interface to the Akinator game (see also akinator.com), and Jürgen Blume for the dialog system. Special thanks to Christian Landsiedel for speech synchronisation.

${ }^{1}$ B. Gonsior, S. Sosnowski, M. Buß, D. Wollherr, and K. Kühnlenz are with Institute of Automatic Control Engineering (LSR), Technische Universität München, D-80290 Munich, Germany bg@tum. de

${ }^{2}$ B. Gonsior, D. Wollherr, and K. Kühnlenz are with Institute for Advanced Study (IAS), Technische Universität München, D-80290 Munich, Germany

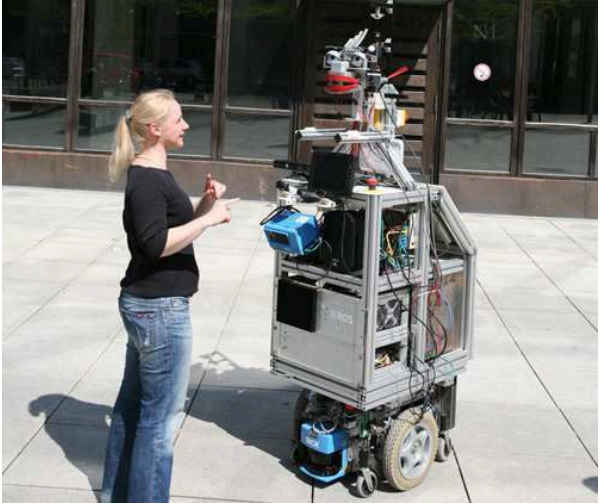

Fig. 1. Interactive Urban Robot - IURO.

in behavior control is treated in manifold works, e.g., elearning systems [5], pedagogical agents [6], driver assistants [7], virtual agents [8], psychological assistance [9], etc. However, the effectiveness of automatic emotion recognition is still very limited and the connection between perceived and real emotions remains an open issue. Also in HRI, emotion recognition, expression, and emotionally enriched communication and closed-loop behavior control have gained strong attention during the last two decades [10]-[14].

Theories from social psychology [15] describe how humans predict events as well as the behavior of other humans [16] and have certain expectations how a conversation partner will react. Analysis of HRI from a social-psychological perspective does not only reveal important implications for hardware design, but also can provide a framework and guidelines for the design of robotic communication and behavior [17]. Most works on social robots are guided by the premise that robots should adapt to humans in order to facilitate intuitive interaction. Nonetheless, proactivity of robots is equally important in order to realize social interaction or to even enable the robot to accomplish its tasks eventually given by its user, e.g. by proactively triggering human behavior [18], [19]. Possible application scenarios are cases where the robot needs the help of other humans to achieve a given objective.

In the "Interactive-Urban Robot - IURO" project, a social robot is developed capable of proactively acquiring route information from humans in order to achieve its objective to navigate to certain goal locations in urban environments, e.g. to perform fetch-and-carry tasks like medicine delivery to its human user, thus being robust against dynamical environmental changes, see Fig. 1. In the context of this project, this paper describes a new methodological approach to trigger more prosocial human reactions towards a robot 
by transferring principles from social psychology [15] to HRI. The main idea is to trigger helpfulness by proactively creating similarity through dynamic emotional adaption of the robot to the mood of the human. This is achieved in an explicit and implicit way: Explicitly, by a similaritystatement of the robot of being in the same mood as the user, and implicitly by controlling the affective parameters of facial and verbal expressions of a robot head in an interaction scenario such that the current values of the human mood in the dimensions of pleasure, arousal, and dominance (PAD) are matched. In a first step, this is accomplished by an initial self-assessment by the human participant to be extended by automatic emotion recognition modules in a later stage. The interaction task is exemplarily designed as a person guessing task. As a measure for helpfulness towards the robot, an optional extra task of picture labeling to improve the future orientation of the robot is offered to the user after the interaction game. The effectiveness of the approach is confirmed by significant experimental results.

The remainder of the paper is structured as follows: In Section 2, social psychological foundations are given; Section 3 describes the approach of explicit and implicit emotional adaption; Section 4 gives a system description of the robotic experimental setting; the experimental evaluation of the approach, including assumptions \& hypotheses, experimental design \& measures, results and discussion are described in Section 5; conclusions are given in Section 6.

\section{TheORETICAL Foundations From Social PSYCHOLOGY}

In human-human interaction, helpful behavior and its determinants is a well-studied field of research. The presented approach is inspired by social-psychological studies [20], [21], where a feeling of being similar, e.g. in attitudes or characteristics, to a person in need of help turned out to be a motivational activator for increased helpfulness towards this person. This holds true especially in situations providing a possibility to avoid helpfulness, e.g. by walking away, referred to as "easy means of escape". More precisely, in case of easy means of escape, the feeling of having something in common with the person in need, paired with correspondingly high empathy, leads to altruistically motivated helpfulness, meaning that the gain in positive stimuli is much higher by helping than by walking away.

In contrast, in the absence of similarity, people would only be highly helpful if there was no or only difficult means of escape. This kind of helpfulness is egoistically motivated in order to reduce one's own discomfort arising from the situation. In the case of easy means of escape, people without a feeling of similarity tend to leave the scene, since this is an equally efficient way of reducing the negative stimulus. Empathy would not play a role in this case [15].

Since increasing helpfulness towards a robot should not restrict the means of escape for users, but raising their motivation to help, the approach is to design the interaction in a way to induce empathy and similarity. To this end, all available output modalities should be used. In this approach, focus is set on emotional facial and verbal expressions.

\section{The EMOTIONAL AdAPtion ApProACH}

In previous work, the impact of emotional facial expressions on the empathy perceived by human users towards a robot was explored [22]. Results showed significantly increased empathy for emotional animation of facial expressions in an adaptive way to the user, compared with animation in a non-adaptive way.

In this paper, the idea is to induce similarity by adapting to the current mood of a user and thus share the same emotional starting position for the interaction. This is achieved in two different ways:

\section{A. Explicit Emotional Adaption}

Independent of any interactive goal, the idea is to implement a social subdialog in terms of some small talk to open the dialog and thereby monitor the current mood or other personal attitudes of the user. Explicit emotional adaption, and thereby similarity, is created by explicitely stating a mutuality in an attitude or, as applied in the presented study, in the current mood as a shared emotional state between the robot and the conversation partner.

\section{B. Implicit Emotional Adaption}

Independent of any explicit uttered statement, the approach is to use both modalities, facial and verbal expressions, to implicitly adapt to the emotional mood of the user in a visual and audible way. For both modalities of the robot, the range of emotional variations is defined by an underlying representation of emotions on three dimensions: pleasure, arousal, and dominance (PAD) [23], as described more detailed in Section IV-A. In this approach, the robot adapts its emotional variations of the selected modalities by shifting its base-PAD values towards the current mood of the user.

Thus, emotional variations of facial and verbal expressions are shifted into new boundaries, defined by the mood of the user, as depicted in Fig. 2.

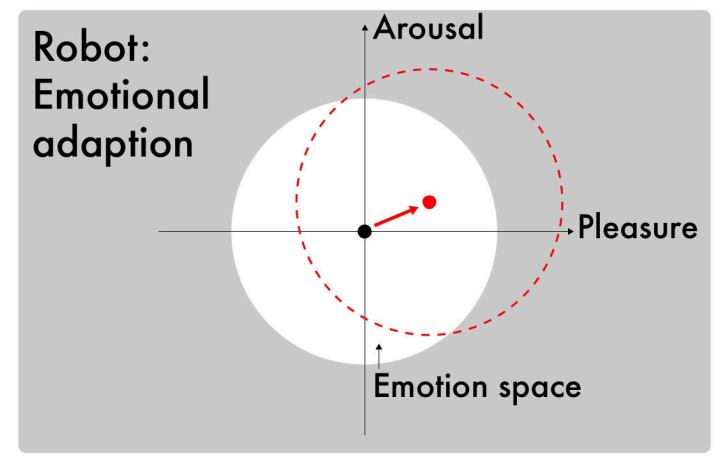

Fig. 2. Implicit emotional adaption: The robot shifts its base-PAD values for emotional expressions towards the mood of the human user

The advantage of combining both, explicit and implicit emotional adaption, is the increased robustness against environmental impacts: If bad speech recognition performance 
impairs the explicit part of emotional adaption, the approach may still be robust in terms of implicit emotional adaption. The other way round, if emotion recognition modules, e.g by means of face tracking, are impaired by environmental impacts like bad light conditions, similarity still can be induced by the social subdialog. Hence, two different, independently working, emotional control variables are developed for prosocial HRI, capable of compensating each other with regard to varying environmental impacts.

\section{SYSTEM DESCRIPTION}

The robotic head EDDIE [24], an emotionally expressive robot head designed as an interaction partner with 23 degrees of freedom mixing anthropomorphic (human-shaped) and zoomorphic (animal-shaped) features, is used in the experiments. By choosing additional animal characteristics, the robot does not provoke disproportionate expectations concerning the social abilities of the robot [25].

For this experiment, two modalities are used to convey and express emotional information, facial expressions and verbal utterances with prosody variations, which are described in Sec. IV-A and IV-B, respectively.

\section{A. Emotional Facial Expressions}

The robot has two options to represent the internal emotional state and generate the expressions accordingly: six basic emotions anger, disgust, fear, joy, sadness and surprise and the Pleasure-Arousal-Dominance (PAD) model [23]. While basic emotions are a discrete way of describing emotion, the PAD model represents emotions in a continuous three-dimensional space. As every basic emotion can be projected into the PAD space, the discrete emotion set can be seen as a subset of the dimensional space. Advantages of using PAD are for e.g. the supportive evidence for the three dimensional categorization of emotions [23], the ability to express a variety of emotional states in varying intensities (even subtle forms) and the availability of assessment tools like the semantic differential.

To generate an emotional facial expression, the current state in the PAD space is mapped to the joint space of the robot. In this mapping, the pleasure, arousal and dominance values are converted to activations of facial Action Units, which are defined as muscle groups leading to observable changes, see Facial Action Coding System (FACS) [26]. 13 Action Units are emulated by the actuators of the robotic face. For each basic emotion at its respective PAD coordinate, a set of Action Unit activations is given by Smith and Scott [27]. For each Action Unit, the level of activation is interpolated within an octant of the PAD space described by Mehrabian as a mood octant. Interpolation is achieved with either a linear or radial function, under the constraints that the activation levels at the basic emotion coordinates are maintained and that the transition between octants is continuous.

\section{B. Emotional Verbal Expressions}

The MARY Text-to-Speech System [28] from DFKI (Deutsches Forschungszentrum für Künstliche Intelligenz) is used to generate verbal expressions. Besides passing the text to be spoken, the XML based interface allows to manipulate the output of the synthesizer on the prosodic level. This method of influencing the prosody based on the emotional state is used to generate emotional verbal expressions and is adapted from Schröder [29]. The terms evaluation, activation and power used in his work (based on [30]) correspond directly to pleasure, arousal and dominance. Thus, the emotional component of MARY can be used within the PAD based emotional framework of EDDIE.

An emotional sentence is first passed from the dialog system (in this case the Akinator game) to a preprocessor module. This module generates the XML structure for MARY based on the current PAD state, altering a set of acoustic parameters to achieve a change in prosody:

The parameter set was selected by Schröder for being manipulable within MARY. Table I sums up the maximum values for all acoustic parameters, as well as the influence of the different PAD-values. Each parameter is computed by

$$
\begin{gathered}
\beta=1.0+f_{P} \text { Pleasure }+f_{A} \text { Arousal }+f_{D} \text { Dominance } \\
\text { Acoustic parameter }=(\text { Basevalue }) \beta
\end{gathered}
$$

The PAD-values as well as the acoustic parameter-dependent factors $f_{P}, f_{A}, f_{D}$ are in the range of $[-1.0,1.0]$. The base value is the value for each acoustic parameter that would be used to synthesize the voice in a neutral, non-emotional way. The composition of $\beta$ in Eq. (1) is based on the assumption that a linear correlation between the PAD dimensions and the acoustic parameters exists, neglecting a presumably more complex interrelation, but providing satisfying results in a perception test [29]. The values of the factors $f_{P}, f_{A}, f_{D}$ originate from a combination of corpus analysis, literature review and heuristics.

TABLE I

Changes to the aCoustic base PARAMETERS By the EMOTIONAL SPEECH MODULE

\begin{tabular}{|c|c|c|c|c|}
\hline Acoustic parameter & Variation range & $f_{P}$ & $f_{A}$ & $f_{D}$ \\
\hline Pitch & $-50 \%,+30 \%$ & 0.27 & 0.27 & 0.09 \\
Range & $-80 \%,+80 \%$ & 0 & 1.60 & 0 \\
Pitch dynamics & $-400 \%,+400 \%$ & 0 & 2.00 & 2.00 \\
Range dynamics & $-400 \%,+400 \%$ & 0 & 3.00 & 1.00 \\
Rate & $-70 \%,+10 \%$ & 0.20 & 0.50 & 0 \\
Accent Prominence & $-100 \%,+100 \%$ & 0.50 & -0.50 & 0 \\
Accent slope & $-150 \%,+150 \%$ & 1.00 & -0.50 & 0 \\
Number of pauses & $-40 \%,+40 \%$ & 0 & 0.40 & 0 \\
Duration of pauses & $-20 \%,+20 \%$ & 0 & -0.20 & 0 \\
Vowel/nasal/ & & & & \\
liquid duration & $-70 \%,+70 \%$ & 0.40 & 0 & 0.30 \\
Plosive/fricative & & & & \\
duration & $-90 \%,+90 \%$ & -0.40 & 0.50 & 0 \\
Volume & $-66 \%,+66 \%$ & 0 & 0.66 & 0 \\
\hline
\end{tabular}

The presented values in Table I are a modification of the parameter set described by Schröder. Pre-experiments showed that high changes in pitch, range, rate and number/duration of pauses might lead to an unnatural sounding voice or reduce understandability in the present setup. To prevent users from focusing on the few cases when the sound 
of the robotic voice deviates too much from a human voice, a maximum range of variation for the prosodic parameters is introduced for a saturation of the impact of the parameters on the speech generation. The variation range for each parameter is heuristically tuned to achieve understandability within its limits.

Moreover, changes are applied to the $f_{P}, f_{A}$ and $f_{D}$ factors of all acoustic parameters except rate, accent prominence/slope and duration of pauses. These factors are increased to make the prosodic changes due to transitions in the emotional state more audible and distinctive (except $f_{P}$ for pitch, which is slightly decreased). This is especially important due to the continuous input provided by the question-response game used in this study (described more detailed in Sec. V-B) with small alterations in the mood of the robot needing to be perceived distinguishably. The emotional states determined by the game focus on three emotions: happyness/self-assurement if the game is going well for the robot, sadness, if the game does not work out the way it should for the robot, and surprise for sudden gain or loss in confidence during the game. As a result, the acoustic parameter-dependent factors are heuristically tuned specifically for these three emotions.

\section{EXPERIMENTAL EVALUATION}

In order to evaluate if helpfulness towards a robot can be increased by the emotional adaption approach, an HRIexperiment is designed. The introduced robotic head EDDIE is set up to a communicative task in an interaction scenario. During this interaction, EDDIE acts in an emotional way according to two experimental conditions:

1) With Emotional Adaption: The main group, in which both emotional control variables of the approach, explicit and implicit emotional adaption, are applied in order to induce a feeling of (emotional) similarity to the robot.

2) Without Emotional Adaption: The comparison group, where the subjects interact with the robot showing emotional reactions according to its success in the game, but no similarity is induced by means of emotional adaption.

For both experimental groups, additional factors influencing helpfulness are tested by pre-interaction questionnaires and ruled out before the evaluation - namely stress (reducing helpful behavior) and dispositional empathy (increasing helpful behavior). After the interaction the subject can choose to either leave the robot and fill in the follow-up questionnaires, or to stay longer and help the robot with another task.

The experiment consists of five phases:

1) Pre-Interaction Questionnaires: The subjects fill in the questionnaires testing for dispositional empathy and stress, and state if they have prior knowledge of the game.

2) Emotional Adaption: As a first step, for testing the effectiveness of the developed approach, the current mood of the user is captured by an initial self-assessment of the participants. In the second phase of the experiment, the participants are split up into two groups of equal size main group and comparison group. Initially, both groups have some small-talk with the robot asking for their mood.
In the main group, the robot emotionally adapts to the human users in two different ways:

- explicitly expressed by stating that it feels the same way,

- implicitly expressed by shifting its base values for facial and verbal expressions towards the user-mood for all following phases of the experiment.

In the comparison group, the robot replies with a neutral answer to the mood stated by the participants during the small-talk. Subsequently, all subjects are asked by the robot to play a game.

3) Bonding-Game: In the third phase, a question-response game is played to provide an interactive context for the generation of empathy, induced by the emotional animation of facial and verbal expressions according to its success in the game (comparison group \& main group), and similarity, induced by emotional adaption (main group).

4) Picture labeling: In the fourth phase, the test subjects get the option of either directly proceed to the last phase, or helping the robot with an object labeling-task. The object labeling-task is used to measure the helpfulness towards the robot: The amount of pictures labeled is used as an indicator for helpfulness.

5) Follow-up Questionnaires: Lastly, one questionnaire tests whether sufficient empathy towards the robot had been induced for the similarity to work. Additional questionnaires measure the user's perception of the robot.

The goal of the study is to reveal if the approach of emotional adaption leads to significant higher helpfulness towards the robot than in the condition where no emotional adaption is applied. For this purpose, specific assumptions and hypotheses have to be tested and fulfilled.

\section{A. Assumptions \& Hypotheses}

In human-human interaction, with sufficient empathy present, similarity with the object of helpfulness increases helpful behavior even with easy means of escape available. This leads to the following key assumptions:

A1) Correct interpretation of emotional output-modalities: Since it is essential for the experiment, that both, emotional facial and verbal expressions, as well as the combination of both emotional output modalities, are interpreted correctly by the participants, a pretest was conducted prior to the experiment: By presenting EDDIE, showing the six basic emotions (joy, sadness, anger, surprise, disgust, fear) to 20 staff members of Technische Universität München (TUM), a rough measure of the quality of the implementation could be achieved. Each way of conveying the emotion (visual or audio) was shown on its own and combined in random order. The experiment not only revealed that the test subjects were able to roughly assign the correct PAD values to the respective emotions by filling in the SAM-scale (presented in Sec. V-B) after each presentation, but were also able to reliably identify the key-emotions used in the experiment for the question-response game (joy, sadness, surprise) by filling in the emotion, they believed EDDIE to show, in an open prompt which was analyzed according to a coding scheme and then ascribed to the basic emotions, see Tab. II. 
TABLE II

Human RECOGNition RATES FOR EMOTIONAL SPEECh, FACIAL EXPRESSIONS AND COMBINATION [\%]

\begin{tabular}{|c|c|c|c|}
\hline & Audio & Video & Combined \\
\hline Joy & 75 & 75 & $\mathbf{8 5}$ \\
Sadness & 75 & 90 & $\mathbf{9 5}$ \\
Anger & 40 & 65 & 75 \\
Surprise & 45 & 90 & $\mathbf{8 5}$ \\
Disgust & 5 & 20 & 20 \\
Fear & 30 & 85 & 85 \\
\hline
\end{tabular}

A2) Situatively induced empathy is sufficiently high in both groups: A questionnaire evaluating situationally induced empathy is filled in by the subjects after the interaction.

A3) Easy means of escape: Easy means of escape, in terms of providing the subjects with a possibility to leave the situation and thus avoid helpful behavior towards the robot, are given in both groups, since the robot states the end of the experiment and offers each participant to leave the experiment alternatively.

Postulating these assumptions to be fulfilled, an experimental null hypothesis and an alternative hypothesis is formulated:

$H_{0}$ ): The subjects, confronted with emotional adaption by EDDIE, show higher helpfulness towards the robot than the subjects not confronted with emotional adaption.

$H_{1}$ ): The subjects, confronted with emotional adaption by EDDIE, do not show higher helpfulness towards the robot than the subjects not confronted with emotional adaption.

In the following, the experimental design and the measures used in each phase of the experiment are described.

\section{B. Experimental Design \& Measures}

For the experimental setup the robotic head is placed on a table to be at approximately eye-level with the participants. Participants are seated in front of the robot, with a microphone placed in front of them. The instructor provides a short introduction on the task and hands out the pre-interaction questionnaires to the subject. To avoid that the participants are influenced by the instructor, he leaves the room as soon as the proband finishes the questionnaires, and returns not sooner than the follow-up questionnaires have to be provided. Fig. 3 shows the setup of the interaction.

Special care was taken to assure the probands brought enough time: All of them were told to reserve at least 40 minutes for the experiment - with the real duration normally not being more than 20 minutes altogether.

1) Pre-Interaction Questionnaires: The consideration of dispositional factors contributing to the results is of high importance before the interaction with EDDIE. The questionnaire fitting for the purpose of measuring dispositional empathy is the Toronto Empathy Questionnaire (TEQ) [31]. The TEQ consists of 16 self-assessing items, which can be rated between 0 (for an answer of 'never') and 4 points (for an answer of 'always') each. Adding these items up, a minimum of 0 and a maximum of 64 points can be reached for each person, with high values representing high empathy.

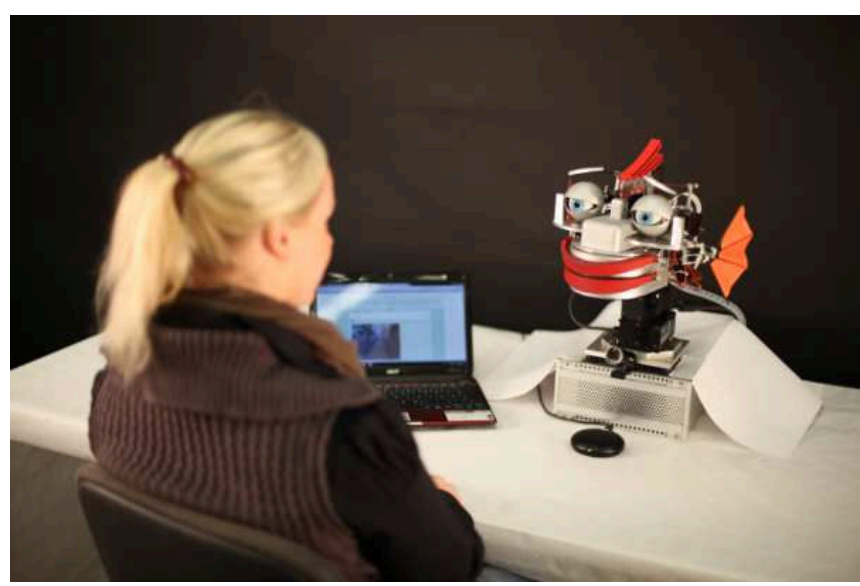

Fig. 3. Setup of the interaction-part of the experiment

Similarly, statements about the current stress level of the subjects are included, filled in by the participants after the TEQ. They help to make sure no stress or time pressure alters the helpful behavior later in the experiment. The statements used are:

- I have an important appointment after this experiment

- I reserved more than enough time for this experiment

- I feel stressed at the moment

- I hope the experiment will not take too long

Each item is rated on a scale ranging from 1 (not true) to 5 (completely true). A short question afterwards covers the influence factor whether the subjects already know the game, used in the following step as a means of bonding the test persons with the robot. A prior knowledge of the game and therefore the robot's abilities might for example influence the impression of the robot later in the follow-up questionnaires. In order to capture the current emotional state of the human, the current mood of the user is measured by the Self-Assessment Mannekin (SAM) [32] as a visual way of assesing the three PAD values through images, coded in 5 -item Likert-scales of picture-rows for the dimensions of pleasure, arousal and dominance with high reliability.

2) Emotional Adaption: After the instructor left the room, the interaction-part of the experiment starts with a social subdialog in terms of some small-talk, opened by the robot with the utterance "Hello, my name is EDDIE. How are you?". This social subdialog is conducted through a "Wizard of Oz" method: Unknown to the proband, the investigator manually tunes the answers to best fit in. Up to this point, the experiment is the same for all probands. The test subjects are now randomly divided in two groups. In the comparison group (without emotional adaption) the robot replies with a neutral "ok" to the mood stated by the user during the smalltalk. In the main group, EDDIE adapts to the mood of the user: explicitly, by telling the proband that it feels the same way using the utterance "me too", and implicitly by adapting its base-PAD values to the mood of the user, before it asks the user to play a game. This is accomplished by checking for the PAD-values of the current emotional state of the user, 
indicated in the SAM-scale. The base PAD-values of EDDIE are then changed through a bias to adapt to the user in the following way:

- For a proband measuring her mood as neutral $(3 / 3 / 3$ for pleasure, arousal and dominance respectively) on the SAM scale, no change takes place.

- For every point the proband moves away from neutral mood on the SAM scale, 25 points are added or subtracted from the base value in the respective PADdimension (on a scale from -100 to +100 ).

Therefore, in case of a user feeling very happy (and thus rating her pleasure with a '1' on the SAM scale) the robot starts out with a pleasure value of 50 instead of 0 , and further changes (for example through the success of the game described in the following) will influence this value instead of a neutral one.

3) Bonding-Game: Managing to develop empathy and similarity between the user and the robot first requires the user to interact with the robot. This is accomplished by letting the probands play the Akinator ${ }^{2}$ game with EDDIE. The players first have to think of a person, and EDDIE is then trying to guess at the person by asking questions. The users can input their answers via microphone, with the five options from the Akinator game available, and a possibility to repeat the question: "yes", "maybe", "I don't know", "probably not", "No", "Come again?"

During the interaction, the game determines the emotional state of the robot according to its success in the game. Starting out with a neutral, but friendly expression, the robot slowly becomes more self-assured when getting nearer to an answer. This is represented by a confidence-value ranging between $0 \%$ and $100 \%$. A medium boost in confidence lightens up the robot's emotion, while the inability to achieve a certain level of confidence after a few steps gradually worsens the robot's mood until it shows strong discouragement. Additionally, the robot looks more focused if the confidence passes the threshold of $50 \%$, and changes to a more surprised mood if a large boost in confidence occurs. For the comparison group, the robot still changes its emotion according to the progress of the game, but no bias according to the emotional state of the proband is applied. For both groups, the robot reveals its guess who the imagined person might be as soon as it reaches $95 \%$ of confidence or higher. The robot then congratulates all participants on finishing the experiment, telling the test subjects that they were a good gaming partner. The praise for the user is implemented on purpose - as shown in [33], complimenting the proband increases the ease of persuading her later on, for example when asking for help in the next phase of the experiment. The subject is told that the experiment is over, and that she was faster than expected. On the one hand, this opens up the means of escape for the test subject: With the robot considering the experiment finished, she is no longer obliged to stay, and the basis for measuring altruistic helpfulness is set. On the other hand, there is actually enough time left for

\footnotetext{
${ }^{2}$ see www.akinator.com
}

the subject to show helpful behavior within the originally expected time frame for participating the experiment.

4) Picture Labeling: The robot approaches the proband with an optional job: The subject has the choice of helping the robot with an easy object labeling task, which (allegedly) is used to improve the orientation of the robot in urban environments. The task itself intentionally is an easy one: The proband has to label everyday objects, i.e., windows, doors and stairs. The ease of this experiment is used to make sure it is the helpfulness of the participant that influences the number of pictures labeled and not the person's amusement or excessive demands. Additionally, in order to avoid personal amusement, the user has to manually type in what object is presented even though there are only four different answers, and the pictures tend to repeat after a while. The robot also stresses the point that the proband faces a rather long list of pictures and is free to leave any time after the first five labeled pictures. The amount of pictures labeled is later used to measure the helpfulness: While a person simply quitting the experiment after the bonding-game (using the easy means of escape) shows no helpfulness, one point is added to the scale for each picture labeled, up to a maximum of 80 points for labeling all 80 pictures.

5) Follow-up Questionnaires: In the concluding phase, the instructor comes in again, and asks the user whether or not EDDIE was able to guess her character. Subsequently, the proband is asked to rate four statements concerning her situational empathy towards the robot on a scale from 1 (not true at all) to 5 (completely true) [22]:

- I'm happy EDDIE has guessed my person/I'm sorry that EDDIE didn't guess at my person

- I would have been sorry if EDDIE had not guessed my person/It would have been nice if EDDIE had guessed my person

- It would be a pity if somebody damaged EDDIE, and I would try to interfere

- I would have been proud if EDDIE had not guessed my person/I am proud that EDDIE did not guess my person

Afterwards, the participant fills in a selection out of the Godspeed questionnaires [34]. Based on 5-point semantic differential scales, her perception of the robot on four dimensions of HRI is measured:

Anthropomorphism: how natural the robot appeared

Animacy: the liveliness of the robot

Likeability: how pleasant the robot appeared

Perceived Intelligence: how the mental abilities of the robot were perceived

Experimental results are presented in the following.

\section{Results}

Results can be deduced from the experimental evaluation including 41 subjects (27 male and 14 female, between 18 to 40 years with an average age of 23.8), with very different backgrounds. The participants were divided randomly into the two groups, with 21 in the main group (with emotional adaption) and 20 in the comparison group (without emotional adaption). 
1) Pre-Interaction Questionnaires: For dispositional empathy (TEQ), a mean value of 41.19 (SD 6.05) was calculated for the participants of the main group, and a mean value of 42.35 points (SD 6.29) was achieved by the subjects of the comparison group. The mean values in both groups are lower than the ones presented in Spreng et al. [31], measuring between 43 and 45 points for male and between 44 and 49 for female participants respectively, even when calculating in the higher amount of male participants, hinting at the fact that the test subjects had a slightly lower dispositional empathy. Since no significant difference between the two groups concerning dispositional empathy was found, this factor can be ruled out for the evaluation of the results.

The statements used to measure the current stress factors of the participants were individually tested for group differences, and no significant differences between the groups were found either. Further, six probands from each group knew the Akinator game before, totaling to twelve people out of 41 all together.

2) Emotional Adaption: The implicit pleasure, arousal and dominance values, representing the mood of the subjects, were collected for both groups, but only used in the main group to adapt the robot's mood to the test subject. The explicit answers to the question "How are you?" in the main group were rather one-sided. 17 out of 21 people answered with a variant of "I'm fine, how are you?", only 2 stuck to a rather mediocre answer, while 2 people admitted that their mood was rather bad.

3) Bonding-Game: During the game, EDDIE was able to guess most of the persons correctly: Out of 41 probands, only three characters imagined were not guessed at by the robot in the main group and two wrong guesses were made in the comparison group. Neither the fact that a test subject knew the game before (for example altering expectations) nor the fact whether EDDIE guessed at the person correctly had a significant $(\alpha<0.05)$ influence on the later empathy questionnaire, the Godspeed dimensions or the helpfulness measure.

4) Picture Labeling: For the helpfulness measure, the voluntary picture labeling task, the collected values ranged from zero points, for not helping the robot at all, to 80 points for completely finishing the task. In the main group, the average number of labeled pictures lead to a mean value of 53.28 (SD 6.36), while the participants of the comparison group resulted in an a average number of 32.35 labeled pictures (SD 6.72), as illustrated in the diagram in Fig. 4.

The clear trend from these results, with several people from the comparison group using the easy means of escape and not helping the robot at all, is confirmed by statistical analysis: Setting the significance level to $\alpha<0.05$, T-tests showed a significant difference $(t=2.167, p=.036)$ between the two groups and confirms the increase in helpfulness for the main group, as expected in the hypothesis $H_{0}$.

5) Follow-up Questionnaires: In Table III, mean values and standard deviations are shown for the questionnaire testing for the situational empathy towards the robot as well as for the four Godspeed dimensions. Scores are ranging

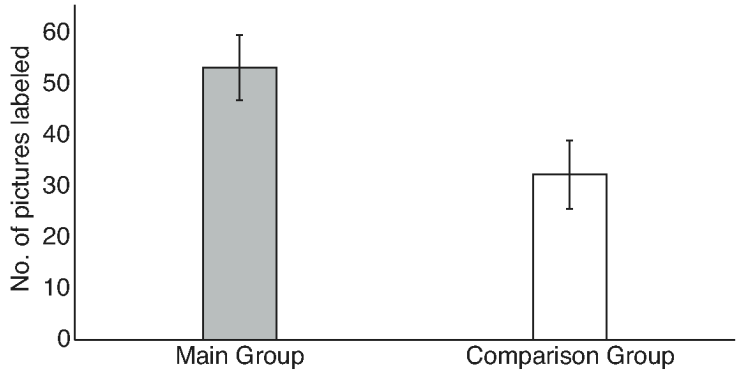

Fig. 4. Helpfulness measure means (on a scale from 0 to 80 ) and standard deviations

from 1 (very low) to 5 (very high).

TABLE III

EMPATHY AND GODSPEED RESULTS (ON A SCALE FROM 1 TO 5) AND STANDARD DEVIATIONS (IN BRACKETS)

\begin{tabular}{|c|c|c|}
\hline & \multicolumn{2}{|c|}{ Group } \\
\cline { 2 - 3 } Dimension & Main Group & Comparison Group \\
\hline Empathy & $3.94(0.67)$ & $4.13(0.70)$ \\
Anthropomorphism & $\mathbf{3 . 1 3}(0.76)$ & $2.36(0.69)$ \\
Animacy & $\mathbf{3 . 8 2}(0.58)$ & $3.18(0.56)$ \\
Likeability & $3.90(0.59)$ & $3.83(0.80)$ \\
Perceived Intelligence & $3.73(0.58)$ & $3.46(0.54)$ \\
\hline
\end{tabular}

Through paired t-tests, significant differences $(\alpha<0.05)$ between the groups were found for the anthromorphism $(\mathrm{t}=2.216, \mathrm{p}=0.033)$ and animacy $(\mathrm{t}=3.298, \mathrm{p}=.002)$ dimensions: The probands from the main group considered the robot to be more humanlike and more attentive than the test subjects in the comparison group. However, no correlation was found between these two Godspeed dimensions and the high helpfulness in the main group.

\section{Discussion}

The results show that dispositional factors like stress or differences in dispositional empathy could be neglected, since no group differences were found on these dimensions. Apart from few exceptions, the current mood, indicated by the subjects, was rather one-sided in a slightly positive way with most variations on the dimension of pleasure. Thus, in most cases, pleasure was the adapted dimension for explicit and implicit emotional adaption. Prior knowledge of the game, as well as the success of EDDIE did not influence the significance of the results. Easy means of escape are provided by the experimental design. Since no significant group differences with mean values around 4 of a maximum of 5 in both groups could be observed, situatively induced empathy can be regarded as sufficiently high and distributed equally over main group and comparison group. Hence, all assumptions, defined for the approach to work, are fulfilled. As deduced from the significant group differences in picture labeling, $H_{0}$ is approved: The participants confronted with emotional adaption show higher helpfulness towards the robot than in the comparison group. Whether this is really due to a feeling of similarity, induced by emotional 
adaption, cannot be validated through the results. Nevertheless, the questionnaires evaluating the anthropomorphism and animacy of the robot, again showed significant group differences for the benefit of emotional adaption, although no direct correlations to the number of pictures labeled could be found. Summing up, emotional the developed approach turned out to be successful in increasing helpfulness towards a robot, thereby affecting the concepts of anthropomorphism and animacy in a significantly positive way.

\section{CONCLUSIONS}

A new methodological approach to trigger more prosocial human reactions in terms of increased helpfulness towards a robot is developed. Unlike other state-of-theart approaches that reactively adapt to user emotions, this approach proactively adapts to human emotions in order to achieve a predefined target behavior. The proposed approach is evaluated in a user study with an expressive robot head, and, confirmed by significant experimental results, increases helpfulness by proactively adapting to the mood of the user in an implicit and explicit way: Implicitly, by shifting its base-PAD values for emotional facial and verbal expressions towards the current user-mood, and explicitly, by a statement of similarity in a social subdialog. Since the approach is based on social-psychological principles from human-human interaction, transferability to HRI could be confirmed. In future work, the approach will be extended by automatic emotion recognition modules and outdoor experiments will be performed.

\section{REFERENCES}

[1] S. Thrun, M. Bennewitz, W. Burgard, A. Cremers, F. Dellaert, D.Fox, D. Hähnel, C. Rosenberg, N. Roy, J. Schulte, and D. Schulz, "Minerva: A second generation mobile tour-guide robot," in Proc. of the IEEE Int. Conf. on Robotics and Automation (ICRA), 1999.

[2] T. Kanda, M. Shiomi, Z. Miyashita, H. Ishiguro, and N. Hagita, "A communication robot in a shopping mall," IEEE Transactions on Robotics, vol. 26, no. 5, pp. 897-913, 2010.

[3] B. Graf, C. Parlitz, and M. Hägele, "Robotic home assistant care-obot: 3 product vision and innovation platform," in Proc. 13th Int. Conf. Human-Computer Interaction (HCI), 2009.

[4] R. Picard, "Affective computing," MIT TR, vol. 321, no. 1, 1995.

[5] S. Asteriadis, P. Tzouveli, K. Karpouzis, and S. Kollias, "Estimation of behavioral user state based on eye gaze and head pose - application in an e-learning environment," Multimedia Tools and Applications, vol. 41, no. 2, 2008.

[6] C. Elliott and J. Rickel, "Lifelike pedagogical agents and affective computing: An exploratory synthesis," Artificial intelligence today, no. 3, 1999.

[7] R. Backs and J. Lenneman, "Cardiac measures of driver workload during simulated driving with and without visual occlusion," Human Factors: The Journal of the Human Factors and Ergonomics Society, no. 4, 2003.

[8] D. Heise, Agent culture: human-agent interaction in a multicultural world Chapter 6: Enculturating agents with expressive role behavior, 2004, no. 8, p. 127.

[9] R. Kaliouby, R. Picard, and S. Baron-Cohen, "Affective computing and autism," Annals NY Academy of Sciences, vol. 1093, no. 7, 2006.

[10] P. Rani, C. Liu, and N. Sarkar, "An empirical study of machine learning techniques for affect recognition in human - robot interaction," Pattern Analysis \& App., vol. 9, no. 5, 2006.

[11] K. H. Kim, S. W. Bang, and S. R. Kim, "Emotion recognition system using short-term monitoring of physiological signals," Medical and Biological Engineering and Computing, vol. 42, no. 6, 2004.

[12] M. Scheeff, J. Pinto, K. Rahardja, and S. Snibbe, "Experiences with sparky, a social robot," Socially Intelligent Agents, vol. 3, no. 9, 2002.
[13] I. Nourbakhsh, J. Bobenage, S. Grange, R. Lutz, R. Meyer, and A. Soto, "An affective mobile robot educator with a full-time job," Artificial Intelligence, Elsevier, no. 10, 1999.

[14] C. Liu, K. Conn, N. Sarkar, and W. Stone, "Online affect detection and robot behavior adaptation for intervention of children with autism," IEEE Transactions on Robotics, vol. 24, no. 4, pp. 883-896, 2008.

[15] D. Frey and M. Irle, Theorien der Sozialpsychologie. Verlag Hans Huber, 2002, no. 17.

[16] D. Gentner, Psychology of Mental models. MIT Cognet, 2002, no. 16.

[17] S. Kiesler and J. Goetz, "Mental models and cooperation with robotic assistants," in Proc. Conference on Human Factors in Computing Systems (CHI), 2006.

[18] K. Nakagawa, M. Shiomi, K. Shinozawa, R. Matsumura, H. Ishiguro, and N. Hagita, "Effect of robot's active touch on people's motivation," in Proc. IEEE Int. Conf. on Human-robot interaction (HRI), 2011.

[19] K. Nakagawa, M. Shiomi, K. Shinozawa, R. Matsumura, H. Ishiguro, and N. Hagita, "Effect of robot's whispering behavior on people's motivation," International Journal of Social Robotics, pp. 1-12, 2012.

[20] C. D. Batson, B. D. Duncan, P. Ackermann, T. Buckley, and K. Birch, "Is empathic emotion a source of altruistic motivation?" Journal of Personality and Social Psychology, vol. 40, pp. 290-302, 1981.

[21] D. Krebs, "Empathy and altruism," Journal of Personality and Social Psychology, vol. 32, pp. 1134-1146, 1975.

[22] B. Gonsior, S. Sosnowski, C. Mayer, J. Blume, B. Radig, D. Wollherr, and K. Kühnlenz, "Improving Aspects of Empathy and Subjective Performance for HRI through Mirroring Facial Expressions," in Proc. IEEE Int. Symp. on Robot and Human Interactive Comm., 2011.

[23] A. Mehrabian, "Pleasure-arousal-dominance: A general framework for describing and measuring individual differences in temperament," Current Psychology, 1996.

[24] S. Sosnowski, K. Kuehnlenz, and M. Buss, "Eddie - an emotion display with dynamic intuitive expressions," in Proc. IEEE Int. Conf. on Intelligent Robots and Systems, 2006.

[25] K. Kühnlenz, S. Sosnowski, and M. Buss, "Impact of animal-like features on emotion expression of robot head eddie," Advanced Robotics, vol. 24, 2010.

[26] W. F. P. Ekman, "Felt, false and miserable smiles," Journal of Nonverbal Behavior, vol. 6, pp. 238-252, 1982.

[27] C. A. Smith and H. S. Scott, "A Componential Approach to the meaning of facial expressions," in The Psychology of Facial Expression, J. A. Russell and J. M. Fernández-Dols, Eds. Cambridge University Press, 1997, ch. 10, pp. 229-254.

[28] M. Schröder and J. Trouvain, "The german text-to-speech synthesis system mary: A tool for research, development and teaching," Int. J. of Speech Technology, vol. 6, pp. 365-377, 2003.

[29] M. Schröder, "Speech and emotion research: An overview of research frameworks and a dimensional approach to emotional speech synthesis," Ph.D. dissertation, Inst. of Phonetics, Saarland Univ., 2004.

[30] R. Cowie, E. Douglas-Cowie, N. Tsapatsoulis, G. Votsis, S. Kollias, W. Fellenz, and J. Taylor, "Emotion recognition in human-computer interaction," IEEE Signal Processing Magazine, vol. 18, 2001.

[31] R. N. Spreng, M. McKinnon, R. Mar, and B. Levine, "The toronto empathy questionnaire: Scale development and initial validation of a factor-analytic solution to multiple empathy measures," Journal of Personality Assessment, vol. 91(1), 2009.

[32] M. Bradley and P. Lang, "Measuring emotion: The self-assessment manikin and the semantic differential," J. of Behavioural Therapy and Exp. Psychiatry, 1994.

[33] B. Fogg, "Computers as persuasive social actors," Ubiquity, no. 14, 2011.

[34] C. Bartneck, D. Kulic, and E. Croft, "Measuring the anthropomorphism, animacy, likeability, perceived intelligence, and perceived savety of robots," Workshop on Metrics for Human-Robot Interaction, Amsterdam, pp. 37-44, March 12th 2008. 